\title{
Reactant preordering solid photopolymers
}

\author{
Anshyang A. Lin, Chi-Fung Chu, Wei-Yu Huang and Arnost Reiser \\ Institute of Imaging Sciences, Polytechnic University, Brooklyn, NY 11201
}

\begin{abstract}
The quantum yield of bimolecular photoreactions in solid polymer films can be enhanced by increasing the fraction of reactive pair sites in the system. A practical realization of this idea is demonstrated on blends of electron donor and electron acceptor substituted poly(vinyl cinnamates). In the triplet sensitized materials energy migration provides an additional method of sensitivity enhancement.
\end{abstract}

The photographic performance of a solid resist film depends not only on the inherent photoreactivity of its components, but also on the immediate environment of the reactants in the solid matrix which may either favor or inhibit the photochemical transformation. This aspect is particularly important in photoprocesses which involve two or more reactants, and where the success of the photoreaction depends on their mutual configuration.

In conventional photopolymers the spatial arrangement of the reactants is a matter of chance and the fraction of reactive configurations is small [1]. In these conditions the number of reactive sites could be significantly enhanced by reactant preordering. We have chosen poly(vinyl cinnamate) as a model system on which to test this idea [2], and we have used electron donor-acceptor interactions as the ordering principle.

Poly(vinyl cinnamate) is a classical resist in which crosslinks are formed by photocycloaddition [3]. In this material the reactive configurations are pairs of cinnamoyl groups in approximately parallel orientation. We thought of increasing the chance of pair formation by bringing together electron donor and electron acceptor substituted cinnamoyl groups in the solid matrix. Several batches of substituted poly(vinyl cinnamates) were prepared [4] as well as similar substituted polymers in which the cinnamoyl groups were uncoupled from the backbone by tri-methylene spacers [5]. These polymers are listed in Table I.

Electron donor and electron acceptor substituted polymers were blended and it was hoped that the blends would show higher photosensitivity than the individual pure components. That was found to be the case, provided the donor-acceptor interactions between the substituted cinnamoyl groups were strong enough. For example, the 50:50 blend of chloro-substituted and methoxy-substituted poly(vinyl

Table I Substituted Poly(vinyl cinnamates)

\begin{tabular}{cc}
$\stackrel{\mathrm{C}}{\mathrm{C}} \mathrm{H}_{2} \cdot \mathrm{O}-\left(\mathrm{CH}_{2}\right)_{n} \mathrm{CO} \cdot \mathrm{CH}=\mathrm{CH}-\bigcirc-\mathrm{O} \quad \mathrm{n}=0$ or 3 \\
\hline $\mathrm{R}$ & $\sigma$ (Hammett) \\
\hline$-\mathrm{OCH}_{3}$ & -0.27 \\
$-\mathrm{CH}_{3}$ & -0.17 \\
$-\mathrm{H}$ & 0 \\
$-\mathrm{Cl}$ & +0.23 \\
$-\mathrm{COOCH}_{3}$ & +0.31 \\
\hline
\end{tabular}


cinnamate) was ten times more photosensitive than the chloro-substituted polymer alone. Photosensitivity was expressed here in terms of the quantum yield of intermolecular crosslink formation, $\Phi$, since intermolecular crosslinks alone contribute to the insolubilitization of the polymer. The quantum yield $\Phi$ of blends of chloro- and methoxy-substituted poly(vinyl cinnamates) as a function of blend composition is shown in Fig.1 . The overall quantum yield, $\phi$, of photocycloaddition (i.e. the quantum yield of intermolecular and intramolecular crosslink formation) is also indicated in the figure.

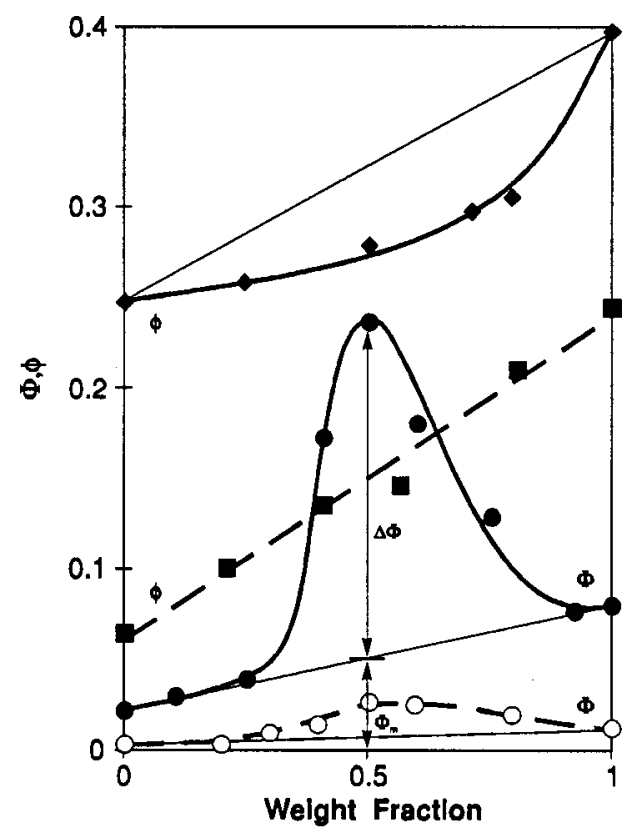

Figure 1 Overall quantum yield $(\phi)$ and quantum yield of intermolecular crosslink formation $(\Phi)$ as a function of blend composition, $\rightarrow$ for chloro- and methoxysubstituted poly(vinyl cinnamates), and $\mathbf{0}$ for the corresponding spacer polymers.

Several conclusions can be drawn from Fig.1.

a) The total number of reactive sites, as measured by the overall quantum yield $\phi$, is not increased in the blends, while the quantum yield of intermolecular crosslink formation $\Phi$ is clearly enhanced. This means that the intramolecular crosslinks, which predominate in the pure polymer, are replaced in the blends by intermolecular ones.

b) The introduction of spacers dilutes the concentration of reactants in the matrix and leads to an overall lowering of the number of reactive pair sites [5].

Seven pairs of substituted polymers were investigated in this study; data on their 50:50 blends are summarized in Table II.

Table II Quantum Yield Change $\Delta \Phi$ and Relative Quantum Yield Change $\Delta \Phi / \Delta \Phi \mathrm{m}$ for Blends of Poly(vinyl cinnamates) and the Corresponding Spacer-Carrying Polymers

\begin{tabular}{ccccccc}
\hline & & & \multicolumn{2}{c}{$\Delta \Phi$} & \multicolumn{2}{c}{$\Delta \Phi / \Delta \Phi_{\mathrm{m}}$} \\
No & substituents & $\Delta \sigma$ & $\mathrm{n}=0$ & $\mathrm{n}=3$ & $\mathrm{n}=0$ & $\mathrm{n}=3$ \\
\hline 1 & $\mathrm{Cl} / \mathrm{AcO}$ & 0.04 & -0.07 & & -0.70 & \\
2,3 & $\mathrm{CH} / \mathrm{H}$ & 0.17 & -0.02 & -0.015 & +0.21 & 0.24 \\
4 & $\mathrm{H} / \mathrm{Cl}$ & 0.23 & +0.032 & & 0.94 & \\
5,6 & $\mathrm{CH}_{3} \mathrm{O} / \mathrm{H}$ & 0.27 & 0.073 & 0.002 & 1.25 & 1.43 \\
7 & $\mathrm{H} / \mathrm{AcO}$ & 0.31 & 0.125 & & 1.90 & \\
8 & $\mathrm{CH}_{3} / \mathrm{Cl}$ & 0.40 & & 0.040 & & 2.54 \\
9 & $\mathrm{CH}_{3} / \mathrm{AcO}$ & 0.48 & 0.14 & & 3.20 & \\
10,11 & $\mathrm{CH}_{3} \mathrm{O} / \mathrm{Cl}$ & 0.50 & 0.18 & 0.050 & 3.50 & 3.58 \\
\hline
\end{tabular}


It was found that in all cases the change in the quantum yield $\Phi$ of intermolecular crosslink formation is directly proportional to the free energy of electron donor-acceptor interaction, as measured by the difference in the Hammet $\sigma$-constants of the substituents. The absolute value of the increase in quantum yield, $\Delta \Phi$, is smaller in the more dilute (spacer) systems (Fig.2), but the relative enhancement of sensitivity is the same in the non-spacer and in the spacer systems. This is demonstrated by the single correlation line of the ratio $\Delta \Phi / \Phi_{\mathrm{m}}$ with $\Delta \sigma$ in the second diagram of Fig.2.. $\Phi \mathrm{m}$ is here the arithmetic mean of the quantum yields of the pure component polymers.
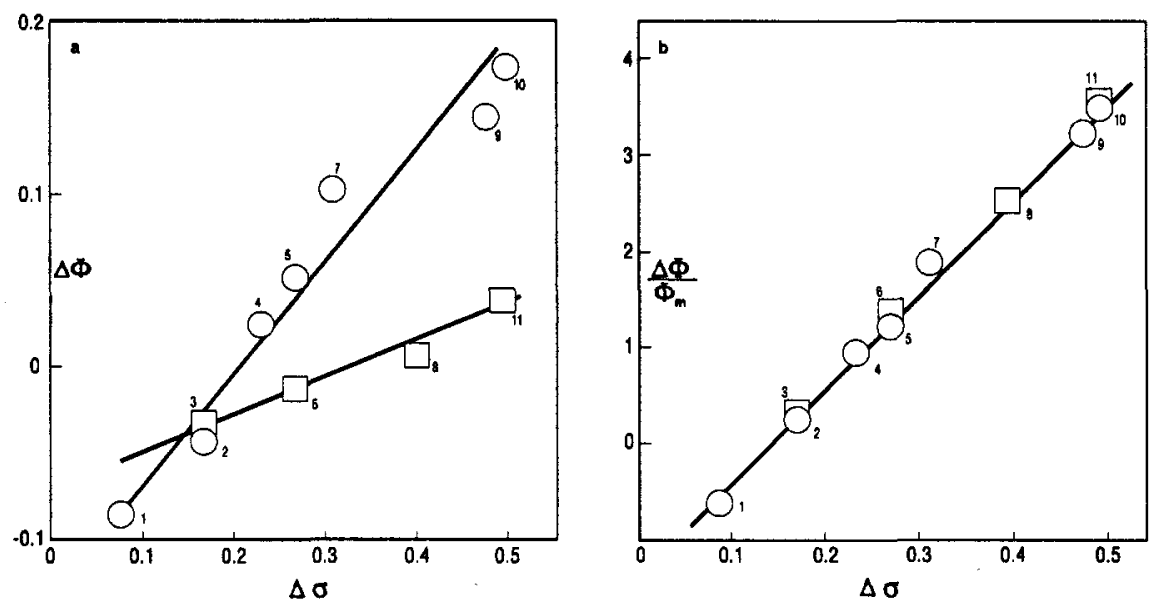

Figure 2 Quantum yield change, $\Delta \Phi$, and relative quantum yield change, $\Delta \Phi / \Phi_{\mathrm{m}}$ for pair-blends of polymers, plotted as a function of the difference in the Hammet $\sigma$-constants of the substituents.

Although the electron donor-acceptor complexes of the substituted cinnamoyl groups cannot be detected by UV spectroscopy, their stabilization energies can be inferred from the effect of the film casting temperature on the quantum yield of crosslink formation. The casting temperature marks the point at which the polymer-solvent system solidifies. From an Arrhenius plot of the data (see Fig.3), a stabilization energy of about $3 \mathrm{kcal} / \mathrm{mol}$ is estimated for a 50:50 blend of chloro-substituted and methoxysubstituted poly(vinyl cinnamate), and a stabilization energy of $7.7 \mathrm{kcal} / \mathrm{mol}$ for the corresponding spacer-carrying system [5].

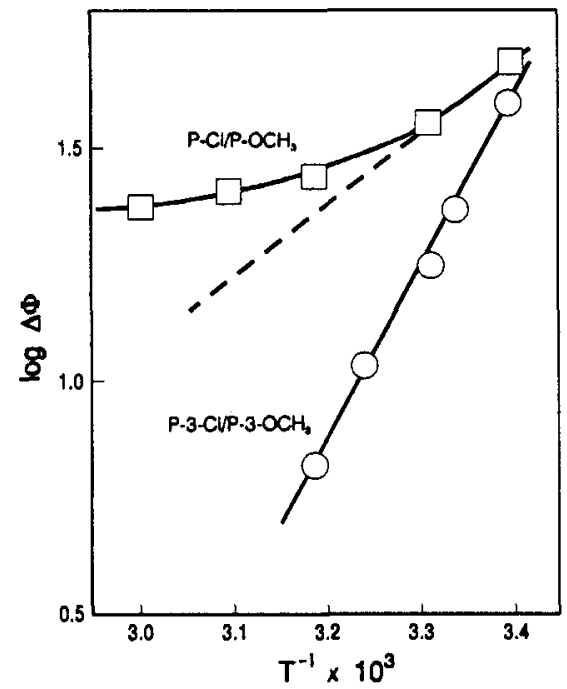

Figure 3 Arrhenius plot of the quantum yield change $\Delta \Phi$ as a function of the casting temperature. 
In the experiments so far described the films were exposed to $300 \mathrm{~nm}$ radiation which is directly absorbed by the substituted cinnamoyl groups. In a second series of experiments [6] the polymers were sensitized by the addition of 3 to $6 \%$ of the triplet sensitizer 3,3'-carbonylbis(coumarin) [7], and exposed to $365 \mathrm{~nm}$ radiation which was absorbed exclusively by the sensitizer. The enhancement of photosensitivity in the triplet sensitized materials was significantly higher than in the unsensitized systems. This is illustrated in Fig.4 which refers to blends of chloro-substituted and methoxy-substituted poly(vinyl cinnamate). A similar, although smaller, additional enhancement was observed with the corresponding spacer polymers.

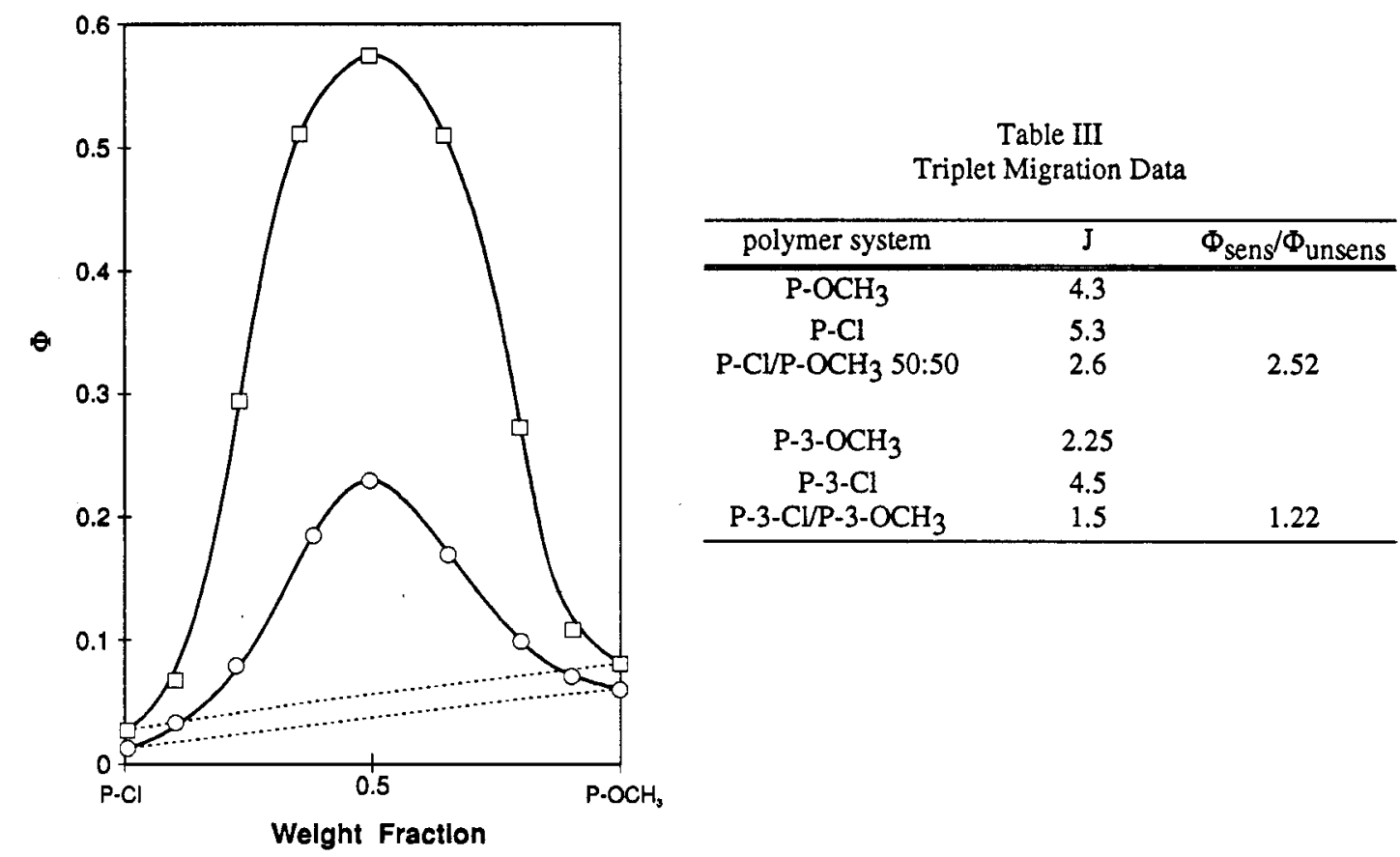

Figure 4 Quantum yield $\Phi$ of intermolecular crosslink formation in blends of chloro- and methoxy-substituted poly(vinyl cinnamates).

$\bigcirc$ unsensitized, $\square$ triplet sensitized.

The effect was traced to the appearance of energy migration in the sensitized systems. The triplet state of the substituted cinnamoyl groups has a long enough lifetime to allow a modest extent of triplet migration in the solid matrix. The singlet excited state of the cinnamoyl group is too short-lived for energy migration to occur. The range of triplet energy migration was measured by a recently described procedure [8] and expressed in terms of the jump number, J, which is the average number of energy transfer steps that the excitation quantum may execute during its lifetime. The results obtained with the chloro- and methoxy-substituted systems and with their 50:50 blends are given in Table III. The following points are of interest here:

a) The migration range in the blends coincides almost exactly with the ratio of the quantum yield of the sensitized to the unsensitizaed system: $\Phi$ (sensitized)/ $\Phi$ (unsensitized).

b) The migration range is shorter in the spacer-carrying systems where the average separation between cinnamoyl groups is larger.

c) The migration range is shorter in the blends than in the pure components. That is caused by the fact that in the blends the reactive sites are electron donor-acceptor complexes and as such have lower 
excitation energies than isolated cinnamoyl groups. They act as energy traps and terminate the migratory path of the excitation quanta at every encounter. In the pure polymers the reactive sites are constituted from identical groups, energy trapping does not occur, and as a consequence the migration range of the excitation is larger.

\section{EXPERIMENTAL PART}

Materials: Substituted poly(vinyl cinnamate) resins were prepared from a single batch of poly(vinyl alcohol), molecular weight 260,000 , (Eastman Kodak) by reacting portions of it in anhydrous pyridine with the acid chlorides of substituted cinnamic acid, in the presence of catalytic quantities of 2,6-di-tertbutylhydroxytoluene (BHT). Besides the compounds mentioned in Table I we prepared also cyanosubstituted poly(vinyl cinnamate), but this was not compatible with any of the other derivatives. The resins were characterized by GPC, by their $\mathrm{Tg}$ values and by their spectra.

The quantum yield of intermolecular crosslink formation: This was derived from the gel dose $\left(\mathrm{E}_{\mathrm{G}}\right)$, the weight average molecular weight $\left(\mathrm{M}_{\mathrm{W}}\right)$ of the polymer, the extinction coefficient $(\varepsilon)$ and the molarity $(\mathrm{m})$ of the radiation absorbing species in the polymer film.

$$
\Phi=\frac{1}{2.303 \varepsilon m M_{W} E_{G}}
$$

The overall quantum yield of the photoreaction: Its value was derived from the evolution of the absorption spectrum of the cinnamoyl chromophore under irradiation.

$$
\phi=\left(\frac{n_{0}}{I_{0} A}\right)\left(\frac{d x}{d t}\right)
$$

where no is the number of chromophores in $1 \mathrm{~cm}^{2}$ of the film, Io is the incident quantum flux (einstein $/ \mathrm{cm}^{2} \mathrm{sec}$ ), $\mathrm{A}$ is the fraction absorbed, $\mathrm{x}$ is the degree of conversion of the cinnamoyl groups and $\mathrm{t}$ is the irradiation time.

Acknowledgement: This work was supported by the National Science Foundation and by the Office of Naval Research of the US Government.

\section{REFERENCES}

1. E. Pitts and A. Reiser, J. Am. Chem. Soc., 105, 5540 (1983).

2. S. Watanabe and $\mathrm{K}$. Ichimura observed an enhancement of sensitivity in blends of substituted poly(vinyl cinnamates) as early as 1982 [J. Polym. Sci., Polym. Chem. Ed. $\underline{20}, 3261$ (1982)].

3. L.M. Minsk, J.G. Smith, W.P. Van Deusen, J.F. Wright, J. Appl. Polym. Sci., 11, 302 (1959).

4. A.A. Lin, A. Reiser, Macromolecules, 22, 3898 (1990).

5. A.A. Lin, C.F. Chu, A. Reiser, Macromolecules, $\underline{23}, 3611$ (1990).

6. W.Y. Huang, A.A. Lin, A. Reiser, Macromolecules, 24, 4600 (1991).

7. D.P. Specht, P.A. Martic, S. Farid, Tetrahedron, 38, 1203 (1982).

8. S.Farid, R.C. Daly, R.E. Moody, W.Y. Huang, A. Reiser, Macromolecules, 24, 4041 (1991). 